\title{
Comparison of machine and manual staining of direct smears for acid-fast bacilli by fluorescence microscopy
}

\author{
J. K. ClANCEY, B. W. Allen, D. T. ROGERS, L. S. SMITH, V. ABER, AND \\ D. A. MITCHISON
}

From the Chest Diseases Laboratory, Lusaka, Zambia and the Medical Research Council's Unit for Laboratory Studies of Tuberculosis, Royal Postgraduate Medical School, London

SYNOPSIS Comparisons were made in Lusaka and in London between manual staining and staining in an automatic machine with auramine-phenol of direct smears of sputum and other types of specimen for acid-fast bacilli. No evidence was obtained of carry-over of acid-fast bacilli from positive to negative smears during machine staining. There was improved contrast between bacilli and the background in smears prepared with the machine.

The staining of direct smears for acid-fast bacilli in Coplin jars is often considered undesirable because of the risk of transferring bacilli from a positive to a negative smear (Pagel et al, 1964). Similar objections could be raised to the use of automatic staining machines. Cremer (1968) investigated the possibility by staining 200 smears prepared from two negative sputum specimens known not to contain acid-fast bacilli, together with a similar number of positive smears prepared from heat-killed cultures of Mycobacterium tuberculosis. No evidence of carryover bacilli from the positive to the negative smears was obtained. However, because of the artificiality of the method of preparation of the positive smears and the rather small samples tested, we felt that a further study was necessary before machine staining could be recommended for general use. We report here the results of two studies, one in Lusaka and the other in London, in which manual and machine staining were compared, particularly to explore the possibility of carry-over from positive to negative smears.

\section{Material and methods}

Separate studies were carried out in Lusaka and in London. In both laboratories, smears of sputum or other types of specimen were spread on slides with a loop made from thick (22 SWG) nichrome wire. A separate slide was used for each specimen. After heat

Received for publication 10 March 1976 fixation, the smears were stained with auraminephenol solution, washed with running water, decolourized with acid-alcohol, washed again, and, in London only, stained with $\mathrm{KMnO}_{4}$ solution to reduce background fluorescence. The staining solutions were prepared as described by Holst et al (1959), except that the auramine-phenol solution contained $3.0 \mathrm{~g}$ auramine $\mathrm{O}$ per litre in both laboratories and the concentration of $\mathrm{HCl}$ in the acidalcohol solution was $0.5 \%(\mathrm{v} / \mathrm{v})$ in Lusaka. Machine staining was done in a Shandon-Elliott 12-station automatic rotating machine (similar to the Varistain 12) which permits 48 slides to be stained in each cycle. The timing of the various stages of staining is set out in table I. In London, staining solutions were replaced daily (which we recommend) to avoid undue evaporation, but weekly in Lusaka. After staining, the smears were examined by fluorescence microscopy using a 200 watt high-pressure mercury vapour bulb, a BG12 excitatory filter, a high numerical aperture bright field condenser, a $\times 25$ objective (giving a total magnification of $\times 250$ in Lusaka and $\times 200$ in London), and an appropriate minus blue barrier filter in the eye piece.

In Lusaka, two smears were made from each of 1286 unselected specimens sent to the laboratory for examination for mycobacteria. Of these, $80 \%$ were sputum and the remainder were body fluids and tissues. One of the pair of smears was stained manually and examined by one of two observers, while the other smear was machine stained and examined by a third observer. Of the 1286 specimens, 


\begin{tabular}{llllll}
\hline Procedure & \multicolumn{3}{l}{ Time taken $(\min )$} & & \\
\cline { 2 - 3 } & \multicolumn{2}{l}{ Lusaka } & & & London \\
\cline { 2 - 3 } \cline { 5 - 6 } & Manual & Machine & & Manual & Machine \\
\hline Auramine & 20 & 20 & & 10 & 4.5 \\
Water wash & 0.5 & 5 & & 0.5 & 1.5 \\
Decolourization & 51 & $5^{1}$ & & 4 & 4.5 \\
Water wash & 0.5 & 5 & & 0.5 & 0.5 \\
Potassium & & & & 0.5 & 1.5 \\
permanganate & - & - & & 0.5 & 1.5 \\
Water wash & - & - & & 16 & 14 \\
Total cycle & 26 & 35 & &
\end{tabular}

Table I Staining procedures

${ }^{1}$ Two separate immersions in acid-alcohol, the first for 2 min and the second for $3 \mathrm{~min}$

$723(56 \%)$ were subsequently cultured on slopes of Löwenstein-Jensen medium after decontamination with $\mathrm{NaOH}$.

In London a group of 227 specimens of sputum likely to contain tubercle bacilli (termed TB POS) were obtained before treatment from Chinese patients in Hong Kong who were participating in a clinical study of short course chemotherapy, their bacteriological assessments being done in London. To gain acceptance to this study, these patients already had had a positive direct smear examination in a Hong Kong laboratory. A further group of 218 specimens unlikely to contain tubercle bacilli (termed TB NEG) were obtained from British patients at Hammersmith Hospital, after the exclusion of any suspected of having tuberculosis. Batches containing similar numbers of TB POS and TB NEG specimens were processed together. A pair of smears was prepared from each specimen and these were randomly allocated to manual or machine staining. After staining, the laboratory number and the mark indicating the staining methods used were covered, the stained smears were again randomly ordered, and were then read by one of three observers. The identity of the smears and the method of staining were uncovered only after the smear had been read and the results recorded. All specimens were cultured by the same method as was used in Lusaka.

\section{Results}

The results of the readings of the smears were very similar whether they were stained manually or by machine (table II). Negative smears were found in $78.2 \%$ of manually stained and in $77.9 \%$ of machine stained smears from 1286 specimens in Lusaka, the corresponding proportions for the 226 TB POS sample in London being $29.6 \%$ and $27.9 \%$, respectively. Despite the high bacterial content of the TB POS specimens, none of the TB NEG specimens yielded smears that were positive for acid-fast 0 bacilli, indicating that the risk of transfer of bacillif during machine staining was very low.

Results in which discrepancies were found between $\Rightarrow$ the manual and machine stained smears are set out $\stackrel{5}{9}$ in table III. Although there were more results

\begin{tabular}{|c|c|c|c|c|c|}
\hline \multirow{4}{*}{$\begin{array}{l}\text { Direct } \\
\text { smear } \\
\text { result }\end{array}$} & \multicolumn{5}{|c|}{ Percentage of smears examined } \\
\hline & \multicolumn{2}{|l|}{ Lusaka } & \multicolumn{3}{|l|}{ London } \\
\hline & \multirow[t]{2}{*}{ Manual } & \multirow[t]{2}{*}{ Machine } & \multicolumn{2}{|c|}{ TB POS } & \multirow{2}{*}{$\begin{array}{l}T B \text { NEG } \\
\text { Manual an } \\
\text { machine }\end{array}$} \\
\hline & & & Manual & Machine & \\
\hline \multirow{5}{*}{$\begin{array}{l}3+ \\
2+ \\
1+ \\
\text { Negative } \\
\text { Total } \\
\quad \text { smears }\end{array}$} & 5.6 & $5 \cdot 5$ & 23.9 & $26 \cdot 1$ & 0 \\
\hline & $3 \cdot 0$ & $2 \cdot 8$ & 15.9 & $13 \cdot 7$ & 0 \\
\hline & $13 \cdot 2$ & $13 \cdot 8$ & $30 \cdot 5$ & $32 \cdot 3$ & 0 \\
\hline & $78 \cdot 2$ & $77 \cdot 9$ & $29 \cdot 6$ & $27 \cdot 9$ & 100 \\
\hline & 1286 & 1286 & 226 & 226 & $218^{1}$ \\
\hline
\end{tabular}

Table II Results of examinations of direct smears stained manually or by machine

${ }^{1}$ On culture: positive, 0 ; negative, 215 ; contaminated, 3

\begin{tabular}{|c|c|c|c|c|c|c|c|}
\hline \multirow[t]{3}{*}{ Laboratory } & \multicolumn{2}{|c|}{ Smear result } & \multirow{2}{*}{\multicolumn{2}{|c|}{$\begin{array}{l}\text { Specimens } \\
\text { examined }\end{array}$}} & \multicolumn{3}{|c|}{ Culture result } \\
\hline & \multirow{2}{*}{ Manual } & \multirow{2}{*}{ Machine } & & & \multirow{2}{*}{$\begin{array}{l}\text { Posi- } \\
\text { tive }\end{array}$} & \multirow{2}{*}{$\begin{array}{l}\text { Nega- } \\
\text { tive }\end{array}$} & $N A$ \\
\hline & & & No. & $\%$ & & & \\
\hline \multirow[t]{2}{*}{ Lusaka } & - & + & 7 & 0.5 & 3 & 2 & 27 \\
\hline & + & - & 3 & 0.2 & 1 & 1 & $1=$ \\
\hline \multirow[t]{2}{*}{ London } & - & + & 7 & $3 \cdot 1$ & 7 & 0 & $0 \frac{0}{7}$ \\
\hline & + & - & 3 & $1 \cdot 3$ & 3 & 0 & $0 \stackrel{?}{?}$ \\
\hline
\end{tabular}

Table III Discrepancies between results on smears stained manually or by machine

NA $=$ not available

positive only in machine stained smears than in manually stained smears both in Lusaka and in. London, the differences were not satistically significant ( $P=0.2$ in both comparisons). All of the specimens with a discrepant result in London? yielded a positive culture for $M$. tuberculosis, bui about half of those in Lusaka, which includedB specimens obtained during chemotherapy and witho long transit periods to the laboratory, yielded no? growth.

Both in Lusaka and in London the observers agreed that the machine stained smears were mores free of nonspecific background fluorescence than then manually stained smears and that the contrasin between the stained bacilli and the background was better. In practice, it was usually easy to distinguish the machine stained smear within a pair. Counter -0 staining with $\mathrm{KMnO}_{4}$ reduced background fluores? cence still further and was disliked in Lusaka but not in London, because it created difficulties in the initial focussing onto the smear. 


\section{Discussion}

No evidence was obtained from either of the studies in Lusaka or in London of transfer of acid-fast bacilli from positive to negative smears during machine staining. Furthermore, the machine-stained smears were cleaner and easier to read. We can therefore recommend machine staining of slides provided that the cost of doing so is justified. We have attempted to calculate the relative costs of manual and machine staining. For 48 slides, a full cycle of manual staining takes about $32 \mathrm{~min}$, of which $10 \mathrm{~min}$ is occupied directly with the handling of slides and stains. The direct handling time for the machine is $2.5 \mathrm{~min}$, so that a saving of about $30 \mathrm{~min}$ is obtained if no other work can be done by a technician during manual staining, because, for instance, staining is done in a separate room. There is a saving of $7.5 \mathrm{~min}$ if, as is unlikely, the technician fully occupies all of the waiting time during manual staining with other technical work. On a basis of an average of 48 slides for 250 days in the year, the time which could be saved by machine staining of a technician paid at $£ 3000$ per annum is then worth between $£ 50$ and $£ 200$. As the cost of a staining machine is about $£ 700$, machine staining is clearly worthwhile in a laboratory with a large workload. It should also be noted that it takes only a few minutes to change the staining pots from those used for auramine staining to those required for Gram staining. A further advantage of machine staining is that it reduces the chances of technical staff getting auramine, which is thought to be a mild carcinogen, on their skin.

We thank the Permanent Secretary, Ministry of Health, Zambia for permission to publish this paper.

\section{References}

Cremer, A. W. F. (1968). Automatic slide staining in clinical bacteriology. J. med. Lab. Technol., 25, 387-390.

Holst, E., Mitchison, D. A., and Radhakrishna, S. (1959). Examination of smears for tubercle bacilli by fluorescence microscopy. Indian J. med. Res., 47, 495-499.

Pagel, W., Simmonds, F. A. H., MacDonald, N., and Nassau, E. (1964). Pulmonary Tuberculosis, 4th ed. Oxford University Press, London. 